\title{
DESCENDING CHAINS OF SEMISTAR OPERATIONS
}

\author{
HYUN SEUNG CHOI, TIMOTHY MCELDOWNEY AND ANDREW WALKER
}

\begin{abstract}
A class of integer-valued functions defined on the set of ideals of an integral domain $R$ is investigated. We show that this class of functions, which we call ideal valuations, are in one-to-one correspondence with countable descending chains of finite type, stable semistar operations with largest element equal to the e-operation. We use this class of functions to recover familiar semistar operations such as the $w$-operation and to give a solution to a conjecture by Chapman and Glaz when the ring is a valuation domain.
\end{abstract}

1. Introduction. Semistar operations were first defined in 1994 by Matsuda and Okabe ([14]) as an extension of the classical star operation, which originated from the work of Krull ([12]) and was formalized by Gilmer ([10]). As a branch of multiplicative ideal theory, star operations have shown to be a capable tool for describing various classes of integral domains and finding new relationships between them. For example, Zafrullah ([23, Theorem 8]) proved that an integral domain $R$ is a Prüfer domain if and only if $R$ is an integrally closed domain such that the $t$-operation and $d$-operation coincide, and Wang and McCasland ([16, Theorem 5.4]) proved that $R$ is a Krull domain if and only if $R$ is a completely integrally closed domain such that the $w$-operation and $v$-operation coincide. In a similar way, semistar operations have received significant interest in the field of multiplicative ideal theory (for instance, see [4], [6], [7], [8], [17], [19], [20], [21], to name only a few).

We investigate here a class of integer-valued functions defined over the set of ideals of a ring, which we call ideal valuations. Their definition is inspired by the properties shared by many of the classical integer-valued functions defined over the ideals of a ring, such as polynomial grade and height (under mild conditions). These ideal valuations can also be constructed naturally from localizing systems, which have been studied extensively (see [2], [4], [9] and [22]). We first use ideal valuations to establish a link to semistar operations and localizing systems. Specifically, we show that each ideal valuation $\nu$ induces a countable descending chain of localizing systems $G(\nu, n)$ and semistar operations $*_{\nu_{n}}$. We show in Theorem 2 in fact that there is a bijection between the set of ideal valuations and the set of countable descending chains of finite type, stable semistar operations with largest operation equal to the e-operation. As an application, we recover in Theorem 5 the $w$-operation as one of the semistar operations in the chain induced by polynomial grade.

We also use ideal valuations to characterize one-dimensional quasi-local domains in Theorem 4 as the domains $R$ where every ideal valuation is constant on proper ideals. As another application, the last section addresses the question raised by Chapman and Glaz ([3, Problem 44]): If $\left\{*_{\alpha}\right\}_{\alpha \in A}$ is a set of semistar operation on $R$, then when is the semistar operation $*_{A}$ defined by $I^{*_{A}}=\bigcap_{\alpha \in A} I^{*_{\alpha}}$ for each $I \in \bar{F}(R)$ of finite type? This question has been considered by Anderson ([1, Theorem 2]) for star operations, and Mimouni ([17, Theorem 2.4]) for semistar operations over conducive domains. We investigate this question, restricted to the case when $R$ is a valuation domain. In particular, we show that when $\left\{*_{\alpha}\right\}_{\alpha \in A}$ form a countable descending chain of stable and finite type semistar operations on a valuation domain, then $*_{A}$ is of finite type precisely when the corresponding ideal valuation has finite range.

Throughout, all rings $R$ are assumed to be integral domains. $K$ will denote the quotient field of $R, \mathcal{S}(R)$ the set of ideals of $R, \mathcal{S}^{\prime}(R)=\mathcal{S}(R)-\{0, R\}$ the set of proper ideals of $R, f \mathcal{S}(R)$ the set of finitely generated ideals of $R$ and $\bar{F}(R)$ the set of nonzero $R$-submodules of $K$. If $I \in \mathcal{S}(R)$, then $V(I)$ will denote the set of prime ideals of $R$ that contain $I$. A quasi-local domain is an integral domain that has only one maximal ideal (not necessarily Noetherian). When we write, $\overline{\mathbb{N}}$, we are referring to the set $\{\infty, 0,1,2,3 \ldots$,$\} .$

1991 AMS Mathematics subject classification. 13A15.

Keywords and phrases. semistar operations, localizing systems, valuation domains. Received by the editors November 14, 2017. 
2. Localizing systems and semistar operations. Recall that a localizing system $\mathcal{F}$ (see $[\underline{4}$, Section $2]$ ) of an integral domain $R$ is a nonempty family of ideals of $R$ such that the following conditions hold:

- (LS1) If $I \in \mathcal{F}$ and $J$ is an ideal of $R$ such that $I \subseteq J$, then $J \in \mathcal{F}$.

- (LS2) If $I \in \mathcal{F}$ and $J$ is an ideal of $R$ such that $\left(J:_{R} i\right) \in \mathcal{F}$ for each $i \in I$, then $J \in \mathcal{F}$.

Furthermore, if for every $I \in \mathcal{F}$, there is some $J \subseteq I$ that's finitely generated and $J \in \mathcal{F}$, then we say that $\mathcal{F}$ is of finite type.

Lemma 1. Let $R$ be a domain, and $S$ a multiplicatively closed subset of $f \mathcal{S}(R)$. If

$$
\mathcal{F}_{S}=\{I \in \mathcal{S}(R) \mid J \subseteq I \text { for some } J \in S\},
$$

then $\mathcal{F}_{S}$ is a finite type localizing system on $R$.

Proof. It's clear that (LS1) holds. We next claim that $\mathcal{F}_{S}$ is multiplicatively closed and is closed under finite intersections. Indeed, if $I, I^{\prime} \in \mathcal{F}_{S}$, then there are $J, J^{\prime} \in S$ with $J \subseteq I$ and $J^{\prime} \subseteq I^{\prime}$, so that $J J^{\prime} \subseteq I I^{\prime} \subseteq I \cap I^{\prime}$, and since $S$ is multiplicatively closed, $J J^{\prime} \in S$. Hence $I I^{\prime}$ and $I \cap I^{\prime}$ are both in $\mathcal{F}_{S}$.

Now we show that $\mathcal{F}_{S}$ satisfies (LS2). Choose ideals $I, J$ of $R$ so that $I \in \mathcal{F}_{S}$ and $\left(J:_{R} i R\right) \in \mathcal{F}_{S}$ for all $i \in I$. We have to show that $J \in \mathcal{F}_{S}$. There exists $I^{\prime} \subseteq I$ with $I^{\prime} \in S$. Let $\left\{i_{k}\right\}$ be a finite generating set of $I^{\prime}$. It follows that $\left(J:_{R} I^{\prime}\right)=\left(J:_{R} \sum i_{k} R\right)=\cap\left(J:_{R} i_{k} R\right)$, so $\left(J:_{R} I^{\prime}\right) \in \mathcal{F}_{S}$ and $I^{\prime}\left(J:_{R} I^{\prime}\right) \in \mathcal{F}_{S}$ by the above claim. Then $J \in \mathcal{F}_{S}$ since $I^{\prime}\left(J:_{R} I^{\prime}\right) \subseteq J$ and by (LS1). Lastly, by definition it follows that $\mathcal{F}_{S}$ is of finite type.

A semistar operation is a map $*: \bar{F}(R) \rightarrow \bar{F}(R)$ such that for any $I, J \in \bar{F}(R)$ and $x \in K \backslash\{0\}$,

(1) $I \subseteq I^{*}$.

(2) $I \subseteq J$ implies $I^{*} \subseteq J^{*}$.

(3) $(x I)^{*}=x I^{*}$.

(4) $\left(I^{*}\right)^{*}=I^{*}$.

Example 1. The following are standard examples of semistar operations:

- The identity operation $d: \bar{F}(R) \rightarrow \bar{F}(R)$ defined by $I_{d}=I$ for all $I \in \bar{F}(R)$.

- The trivial operation $e: \bar{F}(R) \rightarrow \bar{F}(R)$ defined by $I_{e}=K$ for all $I \in \bar{F}(R)$.

- The v-operation $v: \bar{F}(R) \rightarrow \bar{F}(R)$ defined by $I_{v}=\left(R:_{K}\left(R:_{K} I\right)\right)$ for all $I \in \bar{F}(R)$.

- The $t$-operation $t: \bar{F}(R) \rightarrow \bar{F}(R)$ defined by $I_{t}=\bigcup\left\{J_{v} \mid J \subseteq I, J \in f \mathcal{S}(R)\right\}$ for all $I \in \bar{F}(R)$.

- The $w$-operation $w: \bar{F}(R) \rightarrow \bar{F}(R)$ defined by $I_{w}=\bigcup\left\{\left(I:_{K} J\right) \mid J \in f \mathcal{S}(R), J_{v}=R\right\}$ for all $I \in \bar{F}(R)$.

Recall a semistar operation $*: \bar{F}(R) \rightarrow \bar{F}(R)$ is said to be spectral if there is some $\Delta \subseteq \operatorname{Spec}(R)$ so that for any $E \in \bar{F}(R)$,

$$
E^{*}=\bigcap_{P \in \Delta} E R_{P}
$$

In this case, we'll write $*=*_{\Delta}$. We say a semistar operation $*$ is stable if $(I \cap J)^{*}=I^{*} \cap J^{*}$ for all $I, J \in \bar{F}(R)$. Given a semistar operation $*$, define $*_{f}$ such that $I^{*_{f}}=\bigcup\left\{J^{*} \mid J \subseteq I, J \in f \mathcal{S}(R)\right\}$ for all $I \in \bar{F}(R)$. Then $*_{f}$ is a semistar operation, and we say $*$ is of finite type if $*=*_{f}$. Every localizing system $\mathcal{F}$ on a domain $R$ yields a stable semistar operation $*_{\mathcal{F}}$, given as follows ([4, Proposition 2.4]): If $I \in \bar{F}(R)$, then

$$
I^{* \mathcal{F}}=\bigcup_{J \in \mathcal{F}}\left(I:_{K} J\right)
$$


On the other hand, given a semistar operation $*$ on $R$, the set $\mathcal{F}^{*}=\left\{I \in \mathcal{S}(R) \mid I^{*}=R^{*}\right\}$ is a localizing system of $R$ ([4, Proposition 2.8], [4, Remark 2.9]). We adopt the notation $\bar{*}$ for the semistar operation $\mathcal{F}_{\mathcal{F} *}$ and $\tilde{*}$ for the semistar operation $\overline{*_{f}}$. That is, $I^{\bar{*}}=\bigcup\left\{\left(I::_{K} J\right) \mid J \in \mathcal{S}(R), J^{*}=R^{*}\right\}$, $I^{\tilde{*}}=\bigcup\left\{\left(I:_{K} J\right) \mid J \in \mathcal{S}(R), J^{*_{f}}=R^{*}\right\}$ for all $I \in \bar{F}(R)$ (see [5, Section 3]). The theorem below gives a relationship between localizing systems of finite type and semistar operations of finite type.

Theorem 1. [4, Proposition 3.2] Let $\mathcal{F}$ be a localizing system and $*$ a semistar operation defined on $R$.

(1) If $\mathcal{F}$ is of finite type, then $* \mathcal{F}$ is of finite type.

(2) If $*$ is of finite type, then $\mathcal{F}^{*}$ is of finite type.

Lemma 2. Let $R$ be a domain and $S$ a multiplicatively closed subset of $f \mathcal{S}(R)$. Then if $\mathcal{F}_{S}$ is as in Lemma 1. then for any $I \in \bar{F}(R)$,

$$
I^{* \mathcal{F}_{S}}=\bigcup_{J \in \mathcal{F}_{S}}\left(I:_{K} J\right)=\bigcup_{L \in S}\left(I:_{K} L\right) .
$$

Proof. Since $\mathcal{F}_{S}$ is a localizing system, the first equality is by definition of $*_{\mathcal{F}_{S}}$. We now prove the second equality holds. Let $I \in \bar{F}(R)$. Then since $S \subseteq \mathcal{F}_{S}$, obviously

$$
\cup_{L \in S}(I: L) \subseteq \cup_{J \in \mathcal{F}_{S}}(I: J)
$$

Conversely, if $J \in \mathcal{F}_{S}$, then there exists $L^{\prime} \in S$ with $L^{\prime} \subseteq J$. Thus $(I: J) \subseteq\left(I: L^{\prime}\right) \subseteq \cup_{L \in S}(I: L)$, and since this is true for every $J \in \mathcal{F}_{S}$, we have $\cup_{J \in \mathcal{F}_{S}}(I: J) \subseteq \cup_{L \in S}(I: L)$.

\section{Ideal valuation.}

Definition 1. Let $R$ be an integral domain. A function $\nu: \mathcal{S}(R) \rightarrow \overline{\mathbb{N}}$ is an ideal valuation on $R$ if it satisfies the following properties:

- (IV1) $\nu(0)=0$ and $\nu(R)=\infty$.

- (IV2) For any $I, J \in \mathcal{S}(R), \min \{\nu(I), \nu(J)\} \leq \nu(I J)$.

- (IV3) For any $I \in \mathcal{S}(R), \nu(I)=\sup \{\nu(J) \mid J \subseteq I$ and $J \in f \mathcal{S}(R)\}$.

Example 2. If $\mathcal{F}$ is a localizing system of $R$ of finite type, let $\nu_{\mathcal{F}}: \mathcal{S}(R) \rightarrow \overline{\mathbb{N}}$ be the function defined by

$$
\nu_{\mathcal{F}}(I)= \begin{cases}\infty, & \text { if } I=R \\ 1, & \text { if } I \in \mathcal{F} \cap \mathcal{S}^{\prime}(R) \\ 0, & \text { otherwise. }\end{cases}
$$

Then $\nu_{\mathcal{F}}$ is an ideal valuation. Indeed, (IV1) is immediate. Given $I, J \in \mathcal{S}^{\prime}(R), I, J \in \mathcal{F}$ if and only if $I J \in \mathcal{F}$. Therefore $\nu_{\mathcal{F}}(I J) \geq \min \left\{\nu_{\mathcal{F}}(I), \nu_{\mathcal{F}}(J)\right\}$, so that (IV2) is met. If $I \notin \mathcal{F}$, then any ideal contained in $I$ is not an element of $\mathcal{F}$ by (LS1). Hence

$$
\nu_{\mathcal{F}}(I)=\sup \left\{\nu_{\mathcal{F}}(J) \mid J \subseteq I \text { and } J \in f \mathcal{S}(R)\right\}
$$

so that (IV3) holds.

Remark 1. Note that we can't drop the finiteness condition on $\mathcal{F}$ in the previous example. Indeed, let $R$ be a valuation domain whose maximal ideal $M$ is not finitely generated. Then $\{a M \mid a \in R \backslash\{0\}\}$ is the set of nondivisorial ideals of $R$ [5, Proposition 4.2.5]. Thus, in particular, $M$ is nondivisorial and $M_{v}=R$. If $I \neq M$ is a proper ideal of $R$, then $I$ is not principal, so $I$ is not finitely generated and $I_{v}=(a M)_{v}=a M_{v}=a R \neq R$. Hence $\mathcal{F}^{v}=\left\{I \in \mathcal{S}(R) \mid I_{v}=R\right\}=\{R, M\}$. Now $\nu_{\mathcal{F} v}(M)=1$, but then $\nu_{\mathcal{F} v}$ is not an ideal valuation since

$$
\sup \left\{\nu_{\mathcal{F} v}(J) \mid J \subseteq M \text { and } J \in f \mathcal{S}(R)\right\}=0 .
$$


Lemma 3. If $\nu$ is an ideal valuation on $R$, then for $I, I^{\prime} \in \mathcal{S}(R)$ and $I \subseteq I^{\prime}, \nu(I) \leq \nu\left(I^{\prime}\right)$.

Proof. Suppose that $J$ is finitely generated and contained in $I$, then by (IV3), $\nu(J) \leq \nu\left(I^{\prime}\right)$. Taking the supremum over all such $J$ in $I$, by (IV3) again we have $\nu(I) \leq \nu\left(I^{\prime}\right)$.

Lemma 4. If $\nu$ is an ideal valuation on $R$, then for $I, J \in \mathcal{S}(R), \nu(I \cap J)=\nu(I J)=\min \{\nu(I), \nu(J)\}$. In particular, $\nu\left(I^{n}\right)=\nu(I)$ for all $n \geq 1$ and $I \in \mathcal{S}(R)$.

Proof. By (IV2) and the preceding lemma, $\nu(I J) \leq \nu(I \cap J) \leq \min \{\nu(I), \nu(J)\} \leq \nu(I J)$. The second statement follows by letting $J=I^{n-1}$ and a simple induction.

Example 3. If $R$ is a Dedekind domain and $\nu$ an ideal valuation on $R$, then for any $I \in \mathcal{S}^{\prime}(R), \nu(I)$ is completely determined by the value of $\nu$ on maximal ideals of $R$. In fact, since $I=M_{1} M_{2} \cdots$ $M_{n}$ for some maximal ideals $M_{i}$ of $R, \nu(I)=\min _{1 \leq i \leq n}\left\{\nu\left(M_{i}\right)\right\}$.

Lemma 5. Let $R$ be an integral domain and $\nu$ an ideal valuation on $R$. Then given $I \in \mathcal{S}(R), \nu(I)=\nu(\sqrt{I})$.

Proof. By Lemma 3, we have $\nu(I) \leq \nu(\sqrt{I})$. For the other inequality, note that for each $J \in f \mathcal{S}(R)$ such that $J \subseteq \sqrt{I}$, there exists $n \geq 1$ with $J^{n} \subseteq I$. Thus $\nu(J)=\nu\left(J^{n}\right) \leq \nu(I)$ by Lemma 3 and Lemma 4. Hence $\nu(\sqrt{I})=\sup \{\nu(J) \mid J \subseteq \sqrt{I}, J \in f \mathcal{S}(R)\} \leq \nu(I)$.

Lemma 6. If $\nu: f \mathcal{S}(R) \rightarrow \overline{\mathbb{N}}$ satisfies (IV1)-(IV3) on $f \mathcal{S}(R)$, then $\nu$ extends uniquely to an ideal valuation $\widetilde{\nu}$ on $R$.

Proof. Given such a $\nu$, define $\widetilde{\nu}: \mathcal{S}(R) \rightarrow \overline{\mathbb{N}}$ so that $\widetilde{\nu}(I)=\sup \{\nu(J) \mid J \subseteq I$ and $J \in f \mathcal{S}(R)\}$. Then clearly $\widetilde{\nu}$ satisfies (IV1) and (IV3). Now given $I, J \in \mathcal{S}(R)$, suppose that $\widetilde{\nu}(I J)<\min \{\widetilde{\nu}(I), \widetilde{\nu}(J)\}$. Then by (IV3), there are $I^{\prime}, J^{\prime} \in f \mathcal{S}(R)$ so that $J^{\prime} \subseteq J$ and $I^{\prime} \subseteq I$ and

$$
\widetilde{\nu}(I J)<\widetilde{\nu}\left(I^{\prime}\right) \leq \widetilde{\nu}(I) \text { and } \widetilde{\nu}(I J)<\widetilde{\nu}\left(J^{\prime}\right) \leq \widetilde{\nu}(J) .
$$

But then $\widetilde{\nu}(I J)<\min \left\{\widetilde{\nu}\left(I^{\prime}\right), \widetilde{\nu}\left(J^{\prime}\right)\right\} \leq \widetilde{\nu}\left(I^{\prime} J^{\prime}\right)=\nu\left(I^{\prime} J^{\prime}\right) \leq \widetilde{\nu}(I J)$, a contradiction. Thus $\widetilde{\nu}$ satisfies (IV2). Moreover, uniqueness is immediate from (IV3).

Corollary 1. Let $R$ be a domain. If $h: \operatorname{Spec}(R) \rightarrow \overline{\mathbb{N}}$ satisfies $h(0)=0$ and $h(P) \leq h(Q)$ whenever $P \subseteq Q$ in $\operatorname{Spec}(R)$, then $h$ induces an ideal valuation $\widetilde{h}$ on $R$. Explicitly, for any $I \in \mathcal{S}(R)$, we have $\widetilde{h}(I)=\sup \{\inf \{h(P): P \in V(J)\}: J \subseteq I, J \in f \mathcal{S}(R)\}$.

Proof. We have $h$ defines a map $\bar{h}: f \mathcal{S}(R) \rightarrow \overline{\mathbb{N}}$, where for $J \in f \mathcal{S}(R)$,

$$
\bar{h}(J)=\inf \{h(P) \mid P \in V(J)\} .
$$

It's clear that since 0 is a prime ideal, we must have $\bar{h}(0)=0$. By our convention, the infimum of the empty set is $\infty$, so that $\bar{h}(R)=\infty$. Thus $\bar{h}$ satisfies (IV1) on $f \mathcal{S}(R)$. Now suppose $I, J \in f \mathcal{S}(R)$. Then $h(Q)=\bar{h}(I J)$ for some $Q \in \operatorname{Min}(I J)$. Then $Q \in V(I)$ without loss of generality, and so $h(Q) \geq \bar{h}(I)$. Thus (IV2) is met on $\mathcal{S}(R)$. It's clear that (IV3) is satisfied on $f \mathcal{S}(R)$ by the definition of $\bar{h}$ and the monotonicity of $h$, so that by Lemma $6, \bar{h}$ can be extended to an ideal valuation $\widetilde{h}$ on $R$. 
Remark 2. The ideal valuation $\widetilde{h}$ obtained from $h: \operatorname{Spec}(R) \rightarrow \overline{\mathbb{N}}$ above is not necessarily an extension of $h$ without an extra condition on the ring $R$, e.g., if $R$ is Noetherian. Indeed, let $V$ be a valuation domain with value group $\prod_{i=1}^{\infty} \mathbb{Z}$ (under lexicographic ordering). Then for each integer $i \geq 0, V$ has a unique prime ideal $P_{i}$ of height $i$. The maximal ideal $M$ of $V$ is also the unique prime ideal of infinite height. Therefore, for a prime ideal $P$ properly contained in $M$ there exists prime ideals properly between $P$ and $M$. Thus by [10, Theorem 17.3.(e)] $M$ is not the radical of a finitely generated (principal) ideal of $V$. Thus, if we let

$$
h(P)= \begin{cases}0 & P=0 \\ 1 & P \notin\{0, M\} \\ \infty & P=M\end{cases}
$$

then $h(M)=\infty$ but $\widetilde{h}(M)=1$, so that $\widetilde{h}$ is not an extension of $h$.

With the above corollary in mind, for an integral domain $R$, we will say that a function $h: \operatorname{Spec}(R) \rightarrow \overline{\mathbb{N}}$ is a prime valuation on $R$ if it satisfies the following conditions:

(1) $h(0)=0$.

(2) $h(P) \leq h(Q)$ if $P \subseteq Q$.

In other words, $h: \operatorname{Spec}(R) \rightarrow \overline{\mathbb{N}}$ is a prime valuation if and only if $h(0)=0$ and $h$ is a morphism in the category of posets, where $\operatorname{Spec}(R)$ is partially ordered by inclusion.

Lemma 7. Let $R, T$ be domains and $R \rightarrow T$ be a ring homomorphism. If $\nu$ is an ideal valuation on $T$, then $\nu^{c}: \mathcal{S}(R) \rightarrow \overline{\mathbb{N}}$ defined by $\nu^{c}(I)=\nu(I T)$ is an ideal valuation on $R$.

Proof. $\nu^{c}$ clearly satisfies (IV1) since $0 T=0$ and $R T=T$. Let $I, J \in \mathcal{S}(R)$. Then $\nu^{c}(I J)=\nu((I J) T)=$ $\nu(I T J T) \geq \min \{\nu(I T), \nu(J T)\}=\min \left\{\nu^{c}(I), \nu^{c}(J)\right\}$, so that (IV2) holds. Lastly, let $I \in \mathcal{S}(R)$ and $L \in f \mathcal{S}(T)$ so that $L \subseteq I T$. Then $L=\left(\ell_{1}, \ldots, \ell_{n}\right) T$ for some $\ell_{k} \in I T$. So $\ell_{k}=\sum i_{k r} t_{k r}$, where $i_{k r} \in I$ and $t_{k r} \in T$. Let $J=\sum i_{k r} R \in f \mathcal{S}(R)$. Then $L \subseteq J T$, so $\nu(L) \leq \nu(J T)$ by Lemma 3. Since L was arbitrary it follows that $\sup \{\nu(L) \mid L \subseteq I T$ and $L \in f \mathcal{S}(T)\}=\sup \{\nu(J T) \mid J \subseteq I$ and $J \in f \mathcal{S}(R)\}$. Therefore we have $\nu^{c}(I)=\nu(I T)=\sup \{\nu(L) \mid L \subseteq I T$ and $L \in f \mathcal{S}(T)\}=\sup \{\nu(J T) \mid J \subseteq I$ and $J \in f \mathcal{S}(R)\}=\sup \left\{\nu^{c}(J) \mid\right.$ $J \subseteq I$ and $J \in f \mathcal{S}(R)\}$. Thus $\nu^{c}$ is an ideal valuation of $R$.

Lemma 8. Let $R \rightarrow T$ be an inclusion of domains. If $\nu$ is an ideal valuation on $R$, then $\nu^{e}: \mathcal{S}(T) \rightarrow \overline{\mathbb{N}}$, defined by $\nu^{e}(I)=\sup \{\nu(J) \mid J \in f \mathcal{S}(R), J T \subseteq I\}$ for each $I \in \mathcal{S}(T)$, is an ideal valuation on $R$.

Proof. Clearly $\nu^{e}$ satisfies (IV1). Suppose $I_{1}, I_{2} \in \mathcal{S}(T)$. Assume that both $\nu^{e}\left(I_{1}\right)$ and $\nu^{e}\left(I_{2}\right)$ are finite. Then there exist $J_{r} \in f \mathcal{S}(R)$ such that $J_{r} T \subseteq I_{r}$ and $\nu^{e}\left(I_{r}\right)=\nu\left(J_{r}\right)$ for $r=1,2$. Now by Lemma 4 it follows that $\nu^{e}\left(I_{1} I_{2}\right) \geq \nu\left(J_{1} J_{2}\right)=\min \left\{\nu\left(J_{1}\right), \nu\left(J_{2}\right)\right\}=\min \left\{\nu^{e}\left(I_{1}\right), \nu^{e}\left(I_{2}\right)\right\}$. Now assume that only one of $\nu^{e}\left(I_{1}\right)$ and $\nu^{e}\left(I_{2}\right)$ is infinite. Without loss of generality, assume $\nu^{e}\left(I_{1}\right)=\infty$. Choose $L \in f \mathcal{S}(R)$ such that $L T \subseteq I_{2}$ and $\nu^{e}\left(I_{2}\right)=\nu(L)$. Now there exists $J \in f \mathcal{S}(R)$ such that $\nu(J)>\nu(L), J \in f \mathcal{S}(R)$ and $J T \subseteq I_{1}$. Hence by Lemma 4 we have $\nu^{e}\left(I_{1} I_{2}\right) \geq \nu(J L)=\min \{\nu(J), \nu(L)\}=\nu(L)=\nu^{e}\left(I_{2}\right)=\min \left\{\nu^{e}\left(I_{1}\right), \nu^{e}\left(I_{2}\right)\right\}$. Finally, if $\nu^{e}\left(I_{1}\right)=\nu^{e}\left(I_{2}\right)=\infty$, then for each $n \geq 1$ there exists $J_{n}, L_{n} \in f \mathcal{S}(R)$ such that $J_{n} T \subseteq I_{1}, L_{n} T \subseteq I_{2}$ with $\nu\left(J_{n}\right), \nu\left(L_{n}\right)>n$. Thus $\nu^{e}\left(I_{1} I_{2}\right) \geq \nu(J L)=\min \{\nu(J), \nu(L)\}>n$. Since this is true for arbitrary $n \geq 1$, $\nu^{e}\left(I_{1} I_{2}\right)=\infty$. Hence $\nu^{e}$ satisifes (IV2).

It still remains to show that $\nu^{e}$ satisifes (IV3). Suppose that $\nu^{e}(I)=n<\infty$. Note that for each $I_{1}, I_{2} \in \mathcal{S}(T)$, if $I_{1} \subseteq I_{2}$, then $\nu^{e}\left(I_{1}\right) \leq \nu^{e}\left(I_{2}\right)$. Thus it suffices to show that given $I \in \mathcal{S}(R)$, there exists $J \in f S(R)$ such that $J T \subseteq I$ and $\nu^{e}(I) \leq \nu^{e}(J T)$. Now there exists $J \in f \mathcal{S}(R)$ such that $J T \subseteq I$ and $\nu(J)=n$. But then $\nu^{e}(J T)=\sup \{\nu(L) \mid L \in f \mathcal{S}(R), L T \subseteq J T\} \geq \nu(J)=n$. Hence $\nu^{e}(I) \leq \nu^{e}(J T)$, and we're done. For the case when $\nu^{e}(I)=\infty$, given any $n \geq 1$, we have $J_{n} \in f \mathcal{S}(R)$ with $J_{n} T \subseteq I$ such that $\nu\left(J_{n}\right) \geq n$. Then $\nu^{e}\left(J_{n} T\right) \geq n$, so $\nu^{e}(I)=\sup \left\{\nu^{e}(J T) \mid J T \subseteq I, J T \in f \mathcal{S}(T)\right\}=\infty$. Therefore $\nu^{e}$ satisifes (IV3). 
Lemma 9. Let $R \rightarrow T$ be an inclusion of domains. Then the following hold:

(i) If $\nu$ is an ideal valuation on $R$, then $\nu^{e c e}=\nu^{e}$.

(ii) If $\nu$ is an ideal valuation on $T$, then $\nu^{c e c}=\nu^{c}$.

Proof. (i) Note that given $I \in \mathcal{S}(T)$,

$$
\begin{aligned}
\nu^{e c e}(I) & =\sup \left\{\nu^{e c}(J) \mid J \in f \mathcal{S}(R), J T \subseteq I\right\} \\
& =\sup \left\{\nu^{e}(J T) \mid J \in f \mathcal{S}(R), J T \subseteq I\right\} \\
& \leq \sup \left\{\nu^{e}(L) \mid L \in f \mathcal{S}(T), L \subseteq I\right\} \\
& =\nu^{e}(I),
\end{aligned}
$$

On the other hand, for each $J \in f \mathcal{S}(R)$ we have $\nu(J) \leq \nu^{e}(J T)$. So

$$
\begin{aligned}
\nu^{e}(I) & =\sup \{\nu(J) \mid J \in f \mathcal{S}(R), J T \subseteq I\} \\
& \leq \sup \left\{\nu^{e}(J T) \mid J \in f \mathcal{S}(R), J T \subseteq I\right\}=\nu^{e c e}(I),
\end{aligned}
$$

and thus the claim follows.

(ii) Given $I \in \mathcal{S}(R)$,

$$
\begin{aligned}
\nu^{c e c}(I) & =\nu^{c e}(I T) \\
& =\sup \left\{\nu^{c}(J) \mid J \in f \mathcal{S}(R), J T \subseteq I T\right\} \\
& =\sup \{\nu(J T) \mid J \in f \mathcal{S}(R), J T \subseteq I T\} .
\end{aligned}
$$

Since $J T \subseteq I T$, we have $\nu(J T) \leq \nu(I T)$ and so

$$
\begin{aligned}
\nu^{c e c}(I) & =\sup \{\nu(J T) \mid J \in f \mathcal{S}(R), J T \subseteq I T\} \\
& \leq \nu(I T)=\nu^{c}(I) .
\end{aligned}
$$

On the other hand if $J^{\prime} \subseteq I T$ and $J^{\prime} \in f \mathcal{S}(T)$ then there is some $J \in f \mathcal{S}(R)$ such that $J^{\prime} \subseteq J T$. Thus

$$
\begin{aligned}
\nu^{c}(I) & =\nu(I T) \\
& =\sup \left\{\nu\left(J^{\prime}\right) \mid J^{\prime} \subseteq I T \text { and } J^{\prime} \in f \mathcal{S}(T)\right\} \\
& \leq \sup \{\nu(J T) \mid J \in f \mathcal{S}(R), J T \subseteq I T\} \\
& =\nu^{\operatorname{cec}}(I),
\end{aligned}
$$

and so the claim follows.

Example 2 suggests a relationship between localizing systems of finite type and ideal valuations. We'll investigate this further in the following section.

4. Relationship between Localizing Systems and Ideal Valuations. Let $n \geq 0$ and $\nu$ an ideal valuation on a domain $R$. We consider the sets

$$
\widetilde{G}(\nu, n):=\{J \in f \mathcal{S}(R) \mid \nu(J) \geq n\} .
$$

It's easy to see that $\widetilde{G}(\nu, n)$ is multiplicatively closed, so that by Lemma 1 we have an induced localizing system

$$
G(\nu, n):=\mathcal{F}_{\widetilde{G}(\nu, n)} .
$$


In fact, we have that

$$
G(\nu, n)=\{J \in \mathcal{S}(R) \mid \nu(J) \geq n\},
$$

which follows immediately from (IV3). So by our work in the previous section, for every ideal valuation $\nu$ and $n \geq 0$ we have a finite type, stable semistar operation $\nu_{n}: \bar{F}(R) \rightarrow \bar{F}(R)$ given by

$$
I \mapsto I_{\nu_{n}}:=\bigcup_{J \in G(\nu, n)}\left(I:_{K} J\right)=\bigcup_{J \in \widetilde{G}(\nu, n)}\left(I:_{K} J\right)
$$

We'll let $\mathcal{C}_{\nu}$ denote this set $\left\{\nu_{n}\right\}_{n=0}^{\infty}$ of stable, finite type semistar operations.

Lemma 10. Let $R$ be a domain and $\nu$ an ideal valuation on $R$. Then the following hold:

(i) If $n \geq m$, then $\nu_{m} \geq \nu_{n}$.

(ii) $\nu_{0}=e$.

Proof. (i) If $n \geq m$, then $G(\nu, n) \subseteq G(\nu, m)$, so that $\nu_{m} \geq \nu_{n}$. For $(i i)$, just note that $G(\nu, 0)=\mathcal{S}(R)$, so that for any $I \in \bar{F}(R), I_{\nu_{0}}=\bigcup_{J \in \mathcal{S}(R)}\left(I:_{K} J\right)=K$.

By a standard, countable descending chain of finite type, stable semistar operations, we mean a family $\mathcal{C}=\left\{*_{n}\right\}_{n=0}^{\infty}$ of finite type, stable semistar operations where $*_{i} \geq *_{i+1}$ for each $i \geq 0$, where $*_{0}=e$. Such a family $\mathcal{C}$ defines now a function

$$
\begin{aligned}
\nu_{\mathcal{C}}: & \mathcal{S}(R) \rightarrow \overline{\mathbb{N}} \text {, where } \\
& I \mapsto \nu_{\mathcal{C}}(I)=\sup \left\{k \mid I_{*_{k}}=R_{*_{k}}\right\} \text { and } \nu_{\mathcal{C}}(0)=0 .
\end{aligned}
$$

Lemma 11. Suppose $\mathcal{C}$ is a standard, countable descending chain of finite type, stable semistar operations on a domain $R$. Then $\nu_{\mathcal{C}}$ is an ideal valuation on $R$.

Proof. We first observe that since $I_{e}=K=R_{e}$ for any $I \in \mathcal{S}(R)$, it follows that $\nu_{C}(I) \geq 0$. It's clear also that $\nu_{\mathcal{C}}(R)=\infty$, so that (IV1) holds. Suppose now that $I, J \in \mathcal{S}(R)$. We have that $(I J)_{*_{k}}=\left(I_{*_{k}} J_{*_{k}}\right)_{*_{k}}$ for all $k \geq 0$. Say $t=\min \left\{\nu_{C}(I), \nu_{C}(J)\right\}$. Then $(I J)_{*_{k}}=\left(I_{*_{k}} J_{*_{k}}\right)_{*_{k}}=\left(R_{*_{k}} R_{*_{k}}\right)_{*_{k}}=\left(R_{*_{k}}\right)_{*_{k}}=R_{*_{k}}$ for all $k \leq t$, so that $\nu_{\mathcal{C}}(I J) \geq t$. Thus (IV2) is met.

Say now $I \in \mathcal{S}(R)$ and let $t=\nu_{\mathcal{C}}(I)$. If $t<\infty$, then $I_{*_{t}}=R_{*_{t}}$ but $I_{*_{t+1}} \neq R_{*_{t+1}}$. It follows that $1 \in I_{*_{t}}$, so that since $*_{t}$ is of finite type, there is some $J \subseteq I$ that's finitely generated and $1 \in J_{*_{t}}$. Thus $J_{*_{t}}=R_{*_{t}}$. Even more, $J \subseteq I$ implies $J_{*_{t+1}} \subseteq I_{*_{t+1}} \neq R_{*_{t+1}}$, so that $t=\nu_{\mathcal{C}}(J)$. By the same reasoning, for any $J^{\prime} \subseteq I$ finitely generated, $\nu_{\mathcal{C}}\left(J^{\prime}\right) \leq t$, so that $t=\sup \left\{\nu_{\mathcal{C}}(J) \mid J \subseteq I, J \in f \mathcal{S}(R)\right\}$, at least when $t<\infty$. When $t=\infty, 1 \in I_{*_{t}}$ for each $t \geq 1$, so that as in the argument above, there is some $J \subseteq I$ finitely generated with $\nu_{\mathcal{C}}(J)=t$. So $\sup \left\{\nu_{\mathcal{C}}(J) \mid J \subseteq I, J \in f \mathcal{S}(R)\right\}=\infty$. Thus (IV3) holds.

Theorem 2. There is a bijective correspondence $\Psi$ from the set of ideal valuations on $R$ to the set of standard, countable descending chain of finite type, stable semistar operations on $R$, given by $\Psi(\nu)=\mathcal{C}_{\nu}$ with inverse map $\Psi^{-1}$ given by $\Psi^{-1}(\mathcal{C})=\nu_{\mathcal{C}}$.

Proof. Suppose that $\mathcal{C}=\left\{*_{n}\right\}_{n=0}^{\infty}$ is a standard, countable descending chain of finite type, stable semistar operations on $R$. We first claim that $\mathcal{C}=\mathcal{C}_{\nu_{\mathcal{C}}}$, or in other words, that $\left(\nu_{\mathcal{C}}\right)_{n}=*_{n}$ for all $n \geq 0$. Indeed, let $I \in \bar{F}(R)$. Then

$$
I_{\left(\nu_{\mathcal{C}}\right)_{n}}=\bigcup_{J \in G\left(\nu_{\mathcal{C}}, n\right)}\left(I:_{K} J\right) .
$$


Now observe that $G\left(\nu_{\mathcal{C}}, n\right)=\left\{J \in \mathcal{S}(R) \mid \nu_{\mathcal{C}}(J) \geq n\right\}=\left\{J \in \mathcal{S}(R) \mid J_{*_{n}}=R_{*_{n}}\right\}$. Thus we have that

$$
I_{\left(\nu_{\mathcal{C}}\right)_{n}}=\bigcup_{J_{*_{n}}=R_{*_{n}}}\left(I:_{K} J\right)=I_{*_{n}},
$$

where the second equality holds since $*_{n}$ is a stable semistar operation ([4, Remark 2.9] and [4, Theorem 2.10]). Thus we've shown that $\Psi\left(\Psi^{-1}(\mathcal{C})\right)=\mathcal{C}$. On the other hand, suppose that $\nu$ is an ideal valuation on $R$. We must show that $\nu=\nu_{\mathcal{C}_{\nu}}$. So suppose that $I \in \mathcal{S}(R)$ and that $\nu(I)=t$ for some $t \in \overline{\mathbb{N}}$. Then we have that $I \in G(\nu, k)$ if and only if $k \leq t$, which means that $I_{\nu_{k}}=R_{\nu_{k}}$ if and only if $k \leq t$, so that $\nu_{\mathcal{C}_{\nu}}(I)=t$. Thus $\Psi^{-1}(\Psi(\nu))=\nu$.

Let $h$ be a prime valuation on $R$, and for each $n \geq 0$, let

$$
\Delta_{h_{n}}=\{P \in \operatorname{Spec}(R) \mid h(P) \leq n\} .
$$

Then we have a family of spectral semistar operations $\mathcal{C}_{h}=\left\{*_{h_{n}}\right\}_{n=0}^{\infty}$, where $*_{h_{n}}:=*_{\Delta_{h_{n}}}$. Also, $*_{h_{0}} \geq *_{h_{1}} \geq *_{h_{2}} \geq \cdots$, since it's clear that $\Delta_{h_{0}} \subseteq \Delta_{h_{1}} \subseteq \Delta_{h_{2}} \subseteq \ldots$

By a countable descending chain of spectral semistar operations, we mean a family $\mathcal{C}=\left\{*_{k}\right\}_{k=0}^{\infty}$ of spectral semistar operations where $*_{k} \geq *_{k+1}$ for each $k \geq 0$. From such a family, define a function $h_{\mathcal{C}}: \operatorname{Spec}(R) \rightarrow \overline{\mathbb{N}}$ by $h_{\mathcal{C}}(0)=0$, and for $P \in \operatorname{Spec}(R)-\{0\}$,

$$
h_{\mathcal{C}}(P)=\inf \left\{k \mid P^{*_{k}} \neq R^{*_{k}}\right\} .
$$

It's easy to see that $h_{\mathcal{C}}$ is a prime valuation on $R$. Indeed, suppose $P \subseteq Q$ and let $t=h_{\mathcal{C}}(Q)$. Then $P^{*_{t}} \subseteq Q^{*_{t}} \neq R^{*_{t}}$, so that $h_{\mathcal{C}}(P) \leq t$.

We will next establish a bijection between prime valuations and countable descending chains of spectral semistar operations on $R$. First, we require some notation: For a semistar operation $*$ on $R$, let

$$
\Pi^{*}=\left\{P \in \operatorname{Spec}(R)-\{0\} \mid P^{*} \neq R^{*}\right\} .
$$

Lemma 12. [4, Remark 4.9] Let $R$ be a domain, If $*$ is spectral, then $\Pi^{*} \neq \varnothing$ and $I^{*}=\cap\left\{I R_{P} \mid P \in \Pi^{*}\right\}$ for each $I \in \bar{F}(R)$.

Theorem 3. There is a bijective correspondence $\Phi$ from the set of prime valuations on $R$ to the set of countable descending chains of spectral semistar operations on $R$, given by $\Phi(h)=\mathcal{C}_{h}$, with inverse map $\Phi^{-1}(\mathcal{C})=h_{\mathcal{C}}$.

Proof. Let $h$ be a prime valuation on $R$. We must check that $h=h_{\mathcal{C}_{h}}$. That is, we must show that for all $P \in \operatorname{Spec}(R)$,

$$
h(P)=h_{\mathcal{C}_{h}}(P)=\inf \left\{k \mid P^{*_{h_{k}}} \neq R^{*_{h_{k}}}\right\} .
$$

Say $t=h(P)$. Now if $k<t, P \nsubseteq Q$ for any $Q \in \Delta_{h_{k}}$, since otherwise we would have $t=h(P) \leq h(Q) \leq k$. So, when $k<t$,

$$
P^{* h_{k}}=\bigcap_{Q \in \Delta_{h_{k}}} P R_{Q}=\bigcap_{Q \in \Delta_{h_{k}}} R_{Q}=R^{* h_{k}} .
$$

Thus $h_{\mathcal{C}_{h}}(P) \geq t$. If $k=t$, then

$$
P^{*_{h_{t}}}=\bigcap_{Q \in \Delta_{h_{t}}} P R_{Q} \subseteq P R_{P},
$$

since $P \in \Delta_{h_{t}}$, so that since $1 \notin P^{*_{h}}$ we have that $P^{*_{h}} \neq R^{h_{* t}}$, and thus $h_{\mathcal{C}_{h}}(P)=t=h(P)$. So $\Phi^{-1} \Phi(h)=h$ for any prime valuation $h$. 
Conversely, suppose $\mathcal{C}=\left\{*_{k}\right\}_{k=0}^{\infty}$ is a countable descending of spectral semistar operations. We must show that $\mathcal{C}=\mathcal{C}_{h_{\mathcal{C}}}$. That is, for each $k \geq 0$ we need to show $*_{k}=*_{\left(h_{\mathcal{C}}\right)_{k}}$. So suppose $E \in \bar{F}(R)$. By Lemma 12. we have that

$$
E^{*_{k}}=\bigcap_{Q \in \Pi^{* k}} E R_{Q}
$$

while

$$
E^{*\left(h_{\mathcal{C}}\right)_{k}}=\bigcap_{P \in \Delta_{\left(h_{\mathcal{C}}\right)_{k}}} E R_{P} \text { and } \Delta_{\left(h_{\mathcal{C}}\right)_{k}}=\left\{P \in \operatorname{Spec}(R) \mid h_{\mathcal{C}}(P) \leq k\right\}
$$

If $P \in \Delta_{\left(h_{\mathcal{C}}\right)_{k}}$, then $h_{\mathcal{C}}(P) \leq k$. So $P^{*_{t}} \neq R^{*_{t}}$ for some $t \leq k$. Since $*_{t} \geq *_{k}$, we have that $P^{*_{k}} \neq R^{*_{k}}$. Hence $P \in \Pi^{* k}$, and we can conclude that $\Delta_{\left(h_{\mathcal{C}}\right)_{k}} \subseteq \Pi^{*_{k}}$. On the other hand, if $P \in \Pi^{*_{k}}$ and $P \neq 0$, then

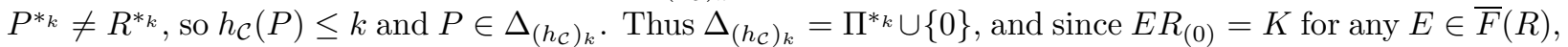
we have $*_{k}=*_{\left(h_{\mathcal{C}}\right)_{k}}$. We conclude that for all $E \in \bar{F}(R), E^{*_{k}}=E^{*_{\left(h_{\mathcal{C}}\right)_{k}}}$, and thus $\Phi \Phi^{-1}(\mathcal{C})=\mathcal{C}$.

We'll say that an ideal valuation $\nu$ is constant on proper ideals of $R$ if there is some $c \in \overline{\mathbb{N}}$ so that for any $I \in \mathcal{S}^{\prime}(R), \nu(I)=c$. Similarly, say that a prime valuation $h$ is constant on nonzero prime ideals of $R$ if there is some $d \in \overline{\mathbb{N}}$ so that for any nonzero prime ideal $P$ of $R, h(P)=d$.

Theorem 4. Let $R$ be an integral domain that is not a field. Then the following are equivalent.

(1) $R$ is a one-dimensional quasi-local domain.

(2) $\tilde{*}=d$ for every semistar operation $* \neq e$ on $R$.

(3) If $*$ is a semistar operation on $R$ that is both stable and of finite type, then either $*=e$ or $*=d$.

(4) Every ideal valuation on $R$ is constant.

(5) Every prime valuation on $R$ is constant and at least one ideal valuation takes a nonzero value.

Proof. (1) $\Leftrightarrow(2)$ : See [21, Theorem 2.8]. (2) $\Leftrightarrow(3)$ : This follows from the fact that $*=\tilde{*}$ if and only if $*$ is stable and of finite type ([4, Corollary 3.9]).

$(3) \Rightarrow(4)$ : Suppose that $d$ and $e$ are the only stable and of finite type semistar operations of $R$. Then by Lemma 10, given an ideal valuation $\nu$ of $R$, either $\nu_{n}=e$ for all $n \geq 0$, or there exists $r \geq 0$ such that $\nu_{n}=e$ for all $0 \leq n \leq r$ and $\nu_{n}=d$ for all $n>r$. Now suppose that $\nu_{n}=e$ for all $n \geq 0$. Then $\nu(I)=\infty$ for all $I \in \mathcal{S}(R) \backslash\{0\}$. Indeed, by Theorem[2, $G(\nu, n)=\left\{J \in \mathcal{S}(R) \mid \nu_{n}(J) \geq n\right\}=\left\{J \in \mathcal{S}(R) \mid J_{v_{n}}=R_{v_{n}}\right\}=\mathcal{S}(R)$, so $\nu(I) \geq n$ for any $I \in \mathcal{S}^{\prime}(R)$. Since this inequality holds for arbitrary $n, \nu(I)=\infty$. Thus $\nu$ is constant on proper ideals. On the other hand, suppose that $\nu_{r}=d$ for some $r \geq 1$ with $r$ chosen minimally. Now by the above argument, $\nu(I) \geq r$ for all $I \in \mathcal{S}^{\prime}(R)$. Assume that $\nu(I)>r$. Then $I \in G(\nu, r+1)$, so that $I_{\nu_{r+1}}=R_{\nu_{r+1}}$, but since $\nu_{r+1}=d$ by assumption, $I=R$. Thus $\nu(I)=r$ for all $I \in \mathcal{S}^{\prime}(R)$, meaning $\nu$ is constant on proper ideals.

$(4) \Rightarrow(2)$ : Suppose that every ideal valuation on $R$ is constant on proper ideals. Let $* \neq e$ be a semistar operation on $R$. Then $\mathcal{F}^{*_{f}}=\left\{I \in \mathcal{S}(R) \mid I^{* f}=R^{*}\right\}$ is a localizing system of finite type by Theorem 1 Set $\nu=\nu_{\mathcal{F}^{*} f}$, as defined in Example 2. Now choose nonzero $x \in R$ such that $x$ is not a unit of $R^{*}$. Then $(x R)^{*_{f}}=x R^{*_{f}} \neq R^{*}$, so $\nu(x R)=0$ and thus $\nu(I)=0$ for all $I \in \mathcal{S}^{\prime}(R)$ by assumption. So $\mathcal{F}^{*_{f}}=\{R\}$ and $I^{\tilde{*}}=I_{\mathcal{F}^{*} f}=I:_{K} R=I$ for all $I \in \bar{F}(R)$, and hence $\tilde{*}=d$.

$(1) \Rightarrow(5)$ : This is immediate, since in this case $\operatorname{Spec}(R)-\{0\}$ consists of a single element, so any prime valuation function $h$ is automatically constant on nonzero prime ideals of $R$. 
$(5) \Rightarrow(1)$ : Suppose that every prime valuation on $R$ is constant on nonzero prime ideals of $R$. Fix $Q \in \operatorname{Spec}(R)-\{0\}$, and define $h: \operatorname{Spec}(R) \rightarrow \overline{\mathbb{N}}$ by

$$
h(P)= \begin{cases}0 & \text { if } Q=0 \\ \infty & \text { if } P \in V(Q) \\ 1 & \text { otherwise }\end{cases}
$$

It's easy to see that $h$ is a prime valuation, so that we must have $V(Q)=\operatorname{Spec}(R)-\{0\}$. Since this holds for any nonzero prime ideal $Q$, we must have that $|\operatorname{Spec}(R)|=2$, meaning $R$ is 1-dimensional quasi-local.

5. Polynomial grade and height. Recall that we say that a sequence $\mathbf{a}=a_{1}, \ldots, a_{r}$ of elements of an arbitrary ring $R$ is a weak $\mathbf{R}$-sequence if for each $i=1, \ldots, r, a_{i} \notin Z\left(R /\left(a_{1}, \ldots, a_{i-1}\right)\right)$, where we write $Z(M)$ to denote the set of zerodivisors of an $R$-module $M$. For an ideal $I$ of $R$, we let grade $(I)$ denote the supremum over all lengths of weak $R$-sequences that are contained in $I$. This is also called the classical grade of $I$ on $R$. In the non-Noetherian setting, classical grade behaves strangely: there are ideals $I$ that consist of zerodivisors, yet $\left(0:_{R} I\right)=0$. Passing to the polynomial ring $R[x]$ can resolve this issue, laying outing out the following notion: The polynomial grade of an ideal $I$ of $R$, denoted by p.grade $(I)$, is the value

$$
\operatorname{p.grade}(I)=\lim _{m \rightarrow \infty} \operatorname{grade}_{R\left[X_{1}, \ldots, X_{m}\right]}\left(I\left[X_{1}, \ldots, X_{m}\right]\right),
$$

where $R\left[X_{1}, \ldots, X_{m}\right]$ denotes the polynomial ring in $m$ variables over $R$.

Lemma 13. Let $I$ be an ideal of an arbitrary ring $R$. Then the following hold:

(1) $\operatorname{grade}_{R}(I) \leq$ p.grade $_{R}(I)$. If $R$ is Noetherian and $I$ is proper, then we have equality.

(2) If $I \neq R$, then there is a prime $\mathfrak{p} \in V(I)$ so that p.grade $(\mathfrak{p})=$ p.grade $(I)$. Moreover, p.grade $(I)=$ p.grade $(\sqrt{I})$.

(3) Let $M$ be an $R$-module and $f \in R\left[X_{1}, \ldots, X_{m}\right]$ and suppose $I$ is generated by the coefficients of $f$. Then $0:_{M} I=0 \Leftrightarrow f$ is a non-zerodivisor on $M\left[X_{1}, \ldots, X_{n}\right]$.

(4) If $J$ is another ideal with $J \subseteq I$, then p.grade $(J) \leq$ p.grade $(I)$.

(5) $p \cdot \operatorname{grade}(I)=\sup \{p \cdot \operatorname{grade}(J) \mid J \subseteq I, J \in f \mathcal{S}(R)\}$.

Proof. These statements, along with their proofs, can all be found in [18, Chapter 5].

Corollary 2. Let $R$ be a domain. Then p.grade: $\mathcal{S}(R) \rightarrow \overline{\mathbb{N}}$ is an ideal valuation on $R$.

Proof. (IV1) and (IV3) follow immediately from the above lemma. Now if $I, J \in \mathcal{S}(R)$ and $t=$ p.grade $(I J)$, then there is some prime ideal $\mathfrak{p}$ of $R$ so that $\mathfrak{p} \in V(I J)=V(I) \cup V(J)$ and p.grade $(\mathfrak{p})=t$. Thus p.grade $(I) \leq t$ or p.grade $(J) \leq t$, and so (IV2) holds.

Thus by Theorem 2, the ideal valuation p.grade yields a family $\left.\{\text { (p.grade })_{n}\right\}_{n=0}^{\infty}$ of finite type semistar operations on $R$. We next relate the (p.grade) ${ }_{n}$ operations to some familiar semistar operations for small values of $n$. First, we need a preparatory lemma.

Lemma 14. Let $J$ be a finitely generated ideal in a domain $R$. Then $J^{-1}=R$ if and only if p.grade $(J) \geq 2$.

Proof. If $J=R$, the claim is trivial, so we can assume throughout that $J$ is proper. So if $J^{-1}=R$, then since $J$ is proper, it's not principal. Thus we may write $J=R b+I$ for some non-zero $b \in J$, where $I \nsubseteq R b$ and $I$ is finitely generated. So write $I=\left(a_{0}, \ldots, a_{n}\right)$, and let $f=a_{0}+a_{1} X+\ldots+a_{n} X^{n} \in R[X]$. We claim that $b, f$ is a $R[X]$-sequence contained in $J[X]$. Clearly $b$ is $R[X]$-regular. Next, we claim that $f$ is a nonzerodivisor on $R[X] / b R[X] \cong(R / b R)[X]$. By Lemma [13(3), this happens precisely when $\left(0:_{R / b R} I\right)=0$. So suppose $r+b R \in(0: R / b R I)$. Then $I r+b R=b R$, so $I r \subset b R \Rightarrow I(r / b) \subset R$. Then $r / b \in J^{-1}=R$, or in other words, $r \in b R$. Hence we have $\left(0:_{R / b R} I\right)=0$, so that $b, f$ forms a $R[X]$-sequence in $J[X]$. 
Thus p.grade $(J) \geq 2$. Conversely, $\operatorname{suppose} \operatorname{p} \operatorname{grade}(J) \geq 2$, so that $\operatorname{grade}_{S}(J S) \geq 2$, where $S$ is a polynomial ring in finitely many variables over $R$. If $\operatorname{grade}_{S}(J S) \geq 2$, then $J S$ contains an $S$-sequence $f, g$. Now if $c \in(J S)^{-1}$, then $c f=t$ and $c g=s$ for some $s, t \in S$. Hence we have $s f=c f g=t g$, which means $t=u f$ for some $u \in S$ since $g$ is a non-zerodivisor on $R / f R$. Thus $c f=t=u f$, which means $c=u \in R$. So $(J S)^{-1}=S$, and hence $J^{-1}=R$.

Theorem 5. Let $R$ be a domain. Then for $i \in\{0,1,2\}$ we have,

$$
(\text { p.grade })_{i}= \begin{cases}e, & \text { if } i=0,1 . \\ w, & \text { if } i=2 .\end{cases}
$$

Proof. First, since p.grade is an ideal valuation, we have (p.grade $)_{0}=e$. Now recall that

$$
G(\text { p.grade, } 1)=\{J \in \mathcal{S}(R) \mid \operatorname{p} \cdot \operatorname{grade}(J) \geq 1\} .
$$

On the other hand, since $R$ is a domain, every nonzero ideal $J$ has p.grade $(J) \geq 1$, so that $G$ (p.grade, 1$)=$ $\mathcal{S}(R)-\{0\}$, and thus

$$
I_{(\text {p.grade })_{1}}=\bigcup_{J \in \mathcal{S}(R)-\{0\}}\left(I:_{K} J\right)=K
$$

for any $I \in \bar{F}(R)$. Thus (p.grade) ${ }_{1}=e$. Lastly, to see (p.grade) $)_{2}=w$, observe by the above lemma we have that

$$
\begin{aligned}
\widetilde{G}(\text { p.grade, } 2) & =\{J \in f \mathcal{S}(R) \mid \operatorname{p.grade}(J) \geq 2\} \\
& =\left\{J \in f \mathcal{S}(R) \mid J^{-1}=R\right\} \\
& =\left\{J \in f \mathcal{S}(R) \mid J_{v}=R\right\} .
\end{aligned}
$$

Thus for any $I \in \bar{F}(R)$, we have that

$$
I_{(\text {p.grade })_{2}}=\bigcup_{J \in \widetilde{G}(\text { p.grade }, 2)}\left(I_{: K} J\right)=\bigcup_{J \in f \mathcal{S}(R), J_{v}=R}\left(I:_{K} J\right)=I_{w} .
$$

Thus (p.grade $)_{2}=w$.

Example 4. Let $R=k[X, Y, Z]$ with $k$ a field and consider the ideal $I=\left(X^{2} Z, Z^{2} Y, Y^{2} X\right)$. As discussed above, p.grade is an ideal valuation on $R$. Now, we have a primary decomposition of $I$ :

$$
I=\left(X^{2}, Y\right) \cap\left(X, Z^{2}\right) \cap\left(Y^{2}, Z\right) \cap\left(X^{2}, Y^{2}, Z^{2}\right) .
$$

Since $R$ is Noetherian, grade and p.grade coincide. Then the primary components have grades $2,2,2,3$ respectively. Thus we have for any $n \geq 1$,

$$
I_{(\text {p.grade })_{n}}= \begin{cases}K & \text { if } n=0,1 \\ R & \text { if } n=2 \\ \left(X^{2}, Y\right) \cap\left(X, Z^{2}\right) \cap\left(Y^{2}, Z\right)=\left(Y Z^{2}, X Y Z, X^{2} Z, X Y^{2}\right) & \text { if } n=3 \\ I & \text { if } n>3\end{cases}
$$

The height function on ideals almost determines an ideal valuation ht: $\mathcal{S}(R) \rightarrow \overline{\mathbb{N}}$. For instance, $\operatorname{ht}(I)=\operatorname{ht}(\sqrt{I})$ for any $I \in \mathcal{S}(R)$, and ht satisfies (IV1) and (IV2). In general though, (IV3) may not be satisfied by the height function. 
Example 5. Let $E$ be the set of entire functions on the complex plane $\mathbb{C}$. The following are well known properties of $E$ (see [10, Exercises 13.16-20]):

(i) $E$ is a non-Noetherian Bézout domain.

(ii) Given $\alpha \in \mathbb{C}, p_{\alpha} E$ is a maximal ideal of $E$ that has height 1 , where $p_{\alpha}: \mathbb{C} \rightarrow \mathbb{C}$ is the entire function that sends $z \mapsto z-\alpha$.

(iii) $\bigcup_{\alpha \in \mathbb{C}} p_{\alpha} E$ is the set of nonunits of $E$.

(iv) $E$ has a prime ideal $P$ that has infinite height.

Now given any finitely generated ideal $J \subseteq P$ of $E, J=a E$ for some $a \in E$ since $E$ is a Bézout domain. Since $a$ is a nonunit, $a \in p_{\alpha} E$ for some $\alpha \in \mathbb{C}$. Hence $\operatorname{ht}(J)=\operatorname{ht}(a E)=\inf \{\operatorname{ht}(M) \mid M \in V(a E)\} \leq \operatorname{ht}\left(p_{\alpha} E\right)=1$, and so $\sup \{h t(J) \mid J \subseteq P, J \in f \mathcal{S}(E)\} \leq 1<\infty=\operatorname{ht}(P)$. Therefore the height function fails to satisfy (IV3).

We will introduce next a fairly weak condition on the ring so that ht: $\mathcal{S}(R) \rightarrow \overline{\mathbb{N}}$ will be an ideal valuation on $R$. A ring $R$ is said to be FGFC (see [13]) if each finitely generated ideal of $R$ has only finitely many minimal primes. We first observe the following property of FGFC rings:

Lemma 15. Let $R$ be a FGFC ring and $I$ an ideal with $h t(I) \geq k$. Then there are $x_{1}, \ldots, x_{k} \in I$ such that $\operatorname{ht}\left(\left(x_{1}, \ldots, x_{i}\right) R\right) \geq i$ for each $i \in\{1, \ldots, k\}$.

Proof. If $k=0$, we have nothing to do, so we proceed by induction on the height of $I$. So suppose that $\operatorname{ht}(I)=k>0$. Now if for every $x \in I$, there is some minimal prime $\mathfrak{p}$ such that $x \in \mathfrak{p}$, then since $R$ is FGFC and prime avoidance, $I$ is contained in some minimal prime, a contradiction. Thus there is some $x_{1} \in I$ such that $x_{1}$ does not lie in any minimal prime $\mathfrak{p}$ of $R$. So ht $\left(x_{1} R\right) \geq 1$.

Now $R x_{1}$ is finitely generated, so that by [13, Proposition 2.2(b)] $R^{\prime}:=R / x_{1} R$ is also a FGFC ring and for $I^{\prime}:=I R^{\prime}$, we must have that $\mathrm{ht}\left(I^{\prime}\right) \geq k-1$. Indeed, if not, suppose that $\mathrm{ht}\left(I^{\prime}\right)<k-1$. Then there is a chain of prime ideals of length $t<k-1$ such that the top prime ideal contains $I^{\prime}: P_{0}^{\prime} \supset \ldots \supset P_{t}^{\prime}$. This lifts to a chain of prime ideals in $R$ that contain $R x_{1}: P_{0} \supset P_{1} \ldots \supset P_{t} \supset R x_{1}$. Now $P_{t}$ cannot be a minimal prime of $R$, hence this chain be extended by one. Since $P_{0} \subseteq I$, we get $\operatorname{ht}(I)<k$, which is a contradiction. Thus by induction, we may choose elements $x_{2}, \ldots, x_{n} \in I$ such that for their images $x_{2}^{\prime}, \ldots, x_{n}^{\prime} \in R^{\prime}$, ht $\left(\left(x_{2}^{\prime}, \ldots, x_{i}^{\prime}\right) R^{\prime}\right) \geq i-1$ for $i=2, \ldots, k$. It then follows by a similar argument as before that $\left(x_{1}, \ldots, x_{i}\right) R$ has height bigger than or equal to $i$.

Corollary 3. Let $R$ be a FGFC domain. Then $\mathrm{ht}: \mathcal{S}(R) \rightarrow \overline{\mathbb{N}}$ is an ideal valuation on $R$.

Proof. As stated earlier, all we must check is (IV3), which follows immediately by the above lemma.

6. A question of Chapman and Glaz. It is well-known that given a collection of overrings $\left\{R_{\alpha}\right\}_{\alpha \in A}$ of a domain $R$, if $*_{\alpha}$ is a semistar operation on $R_{\alpha}$ for each $\alpha \in A$, then the map $*_{A}: \bar{F}(R) \rightarrow \bar{F}(R)$ defined by $I^{*_{A}}=\cap_{\alpha \in A}\left(I R_{\alpha}\right)^{*_{\alpha}}$ for each $I \in \bar{F}(R)$ is a semistar operation ([4, Example $\left.1.3(\mathrm{~d})\right]$ ). In this section, we wish to investigate the following problem posed in [3, Problem 44]: Find conditions for $*_{A}$ to be of finite type, or equivalently, if $\left\{*_{\alpha}\right\}_{\alpha \in A}$ is a set of semistar operation on $R$, then when is the semistar operation $*_{A}$ defined by $I^{*_{A}}=\bigcap_{\alpha \in A} I^{*_{\alpha}}$ for each $I \in \bar{F}(R)$ of finite type?

We will consider this question under the assumption that $R$ is a valuation domain, or in other words, when $\bar{F}(R)$ is totally ordered under inclusion. In this scenario, every semistar operation is stable. Indeed, given $I, J \in \bar{F}(R)$ in a valuation domain $R, I \subseteq J$ without loss of generality, so $(I \cap J)^{*}=I^{*}=I^{*} \cap J^{*}$ for each semistar operation $*$ on $R$. From this observation, we have the following lemma: 
Lemma 16. Let $R$ be a domain and $\left\{*_{\alpha}\right\}_{\alpha \in A}$ a set of semistar operations on $R$. Then the following hold:

(1) $\mathcal{F}^{*_{A}}=\cap_{\alpha \in A} \mathcal{F}^{*_{\alpha}}$.

(2) If every semistar operation on $R$ is stable, then $*_{A}$ is of finite type if and only if $\cap_{\alpha \in A} \mathcal{F}^{*_{\alpha}}$ is of finite type.

Proof. (1): Note that $\left(I^{*_{A}}\right)^{*_{\beta}}=I^{*_{\beta}}$ for each $I \in \bar{F}(R)$ and $\beta \in A$ by ([4, Proposition 1.6 (4)]). Therefore,

$$
\begin{aligned}
I \in \mathcal{F}^{*_{A}} & \Leftrightarrow I^{*_{A}}=R^{*_{A}} \\
& \Leftrightarrow I^{*_{\alpha}}=R^{*_{\alpha}} \text { for each } \alpha \in A \\
& \Leftrightarrow I \in \mathcal{F}^{*_{\alpha}} \text { for each } \alpha \in A \\
& \Leftrightarrow I \in \cap_{\alpha \in A} \mathcal{F}^{*_{\alpha}} .
\end{aligned}
$$

(2): Given a semistar operation $*$ on $R, *$ is of finite type if and only if $\mathcal{F}^{*}$ is of finite type, by our assumption, along with Theorem 1 and [4, Theorem 2.10(A)]. Now by (1), $*_{A}$ is of finite type if and only if $\mathcal{F}^{*_{A}}$ is of finite type if and only if $\cap_{\alpha \in A} \mathcal{F}^{*_{\alpha}}$ is of finite type.

Lemma 17. Let $R$ be a valuation domain. Then the following statements hold:

(1) (19, Lemma 2.40]) If $*$ is a finite type semistar operation on $R$, then there is an overring $T$ of $R$ so that $I^{*}=I T$ for each $I \in \bar{F}(R)$.

(2) (15, Theorem 10.1]) Each overring of $R$ is of the form $R_{P}$, where $P \in \operatorname{Spec}(R)$.

Corollary 4. Let $R$ be a valuation domain and $*$ a semistar operation on $R$. Then the following are equivalent:

(1) * is of finite type.

(2) For some $P \in \operatorname{Spec}(R), I^{*}=I R_{P}$ for each $I \in \bar{F}(R)$.

(3) There is an overring $T$ of $R$ so that $I^{*}=I T$ for each $I \in \bar{F}(R)$.

From this, we obtain a characterization of localizing systems of finite type on a valuation domain.

Lemma 18. Let $R$ be a valuation domain and $\mathcal{F}$ a localizing system of $R$. Then $\mathcal{F}$ is of finite type if and only if $\mathcal{F}=\{I \in \mathcal{S}(R) \mid P \subsetneq I\}$ for some prime ideal $P$ of $R$.

Proof. Suppose that $\mathcal{F}$ is of finite type. Note first that $*_{\mathcal{F}}$ is a semistar operation of finite type on $R$ by Theorem 1. Thus by Corollary 4 there is $P \in \operatorname{Spec}(R)$ with $I^{*_{\mathcal{F}}}=I R_{P}$ for each $I \in \bar{F}(R)$. Now, by [4, Theorem 2.10(A)], we have

$$
\begin{aligned}
\mathcal{F} & =\left\{I \in \mathcal{S}(R) \mid I^{* \mathcal{F}}=R^{* \mathcal{F}}\right\} \\
& =\left\{I \in \mathcal{S}(R) \mid I R_{P}=R_{P}\right\} \\
& =\{I \in \mathcal{S}(R) \mid I \nsubseteq \nexists P\} \\
& =\{I \in \mathcal{S}(R) \mid P \subsetneq I\} .
\end{aligned}
$$

Conversely, say $\mathcal{F}=\{I \in \mathcal{S}(R) \mid Q \subsetneq I\}$ for some $Q \in \operatorname{Spec}(R)$. Then given $I \in \mathcal{F}$, choose $x \in I \backslash Q$. Since $R$ is a valuation domain, $Q \subsetneq x R \subseteq I$ and $x R \in \mathcal{F}$. Therefore $\mathcal{F}$ is of finite type. 
Lemma 19. Let $R$ be a valuation domain, and $\nu$ an ideal valuation of $R$. Then

$$
\operatorname{dim}(R)+1 \geq \#\{\nu(I) \mid I \in \mathcal{S}(R)\} .
$$

Proof. For each $n>0$, let $P_{n}=\{x \in R \mid \nu(x R)<n\}$. First, we show that $P_{n}$ is an ideal of $R$. Indeed, let $x, y \in P_{n}$ and say $r \in R$. Without loss of generality, we have $x R \subseteq y R$, so that by monotonicity of $\nu$, it follows that $\nu((x+y) R) \leq \nu(x R+y R)=\nu(y R)<n$. Hence $x+y \in P_{n}$. Also by monotonicity of $\nu$, we have $\nu((r x) R) \leq \nu(x R)<n$, and so $r x \in P_{n}$. Thus each $P_{n}$ is an ideal of $R$. Now suppose $I, J$ are ideals of $R$ with $I J \subseteq P_{n}$. Then either $I \subseteq P_{n}$ or $J \subseteq P_{n}$ by (IV2). Thus for each $n>0$ we have $P_{n} \in \operatorname{Spec}(R)$, and since the $\left\{P_{n}\right\}_{n=1}^{\infty}$ form an ascending chain, the claim follows.

Lemma 20. If $\nu$ is an ideal valuation on a valuation domain $R$, then $\nu$ has finite range if and only if $\cap_{n \geq 0} G(\nu, n)$ is a localizing system of $R$ of finite type.

Proof. If $\cap_{n \geq 0} G(\nu, n)$ is a localizing system of $R$ of finite type, then by Lemma 18, there is $P \in \operatorname{Spec}(R)$ so that $\nu(I)=\infty$ if and only if $P \subsetneq I$. In particular, $\nu(P)=m$ for some $m \geq 0$. So, for any $I \in \mathcal{S}(R)$, either $\nu(I) \leq m$ or $\nu(I)=\infty$. Therefore $\nu$ has finite range. On the other hand, if the range of $\nu$ is finite, then there is $m \geq 0$ so that $G(\nu, m)=\cap_{n \geq 0} G(\nu, n)$.

Theorem 6. Let $R$ be a valuation domain, $\left\{*_{\alpha}\right\}_{\alpha \in A}$ a set of semistar operations on $R$. Then the following are equivalent.

(1) $*_{A}$ is of finite type.

(2) $\cap_{\alpha \in A} \mathcal{F}^{*_{\alpha}}$ is of finite type.

(3) There exists $\alpha \in A$ such that $I^{*_{A}}=I R^{*_{\alpha}}$ for each $I \in \bar{F}(R)$.

Proof. (1) $\Leftrightarrow(2)$ : This follows from Lemma 16 (1) $\Rightarrow(3)$ : Note that for each $\alpha \in A$, there is some $P_{\alpha} \in \operatorname{Spec}(R)$ such that $R^{*_{\alpha}}=R_{P_{\alpha}}$ by Lemma 17. Suppose that $*_{A}$ is of finite type. Then by Corollary 4 . there is $P \in \operatorname{Spec}(R)$ with $I^{*_{A}}=I R_{P}$ for each $I \in \bar{F}(R)$. It follows $P_{\alpha} \subseteq P$ for each $\alpha \in A$. If $P_{\alpha} \subsetneq P$ for each $\alpha \in A$, then $P^{*_{A}}=\cap_{\alpha \in A} P^{*_{\alpha}} \supseteq \cap_{\alpha \in A} P R_{P_{\alpha}}=\cap R_{P_{\alpha}}=R^{*_{A}}=R_{P} \supsetneq P R_{P}=P^{*_{A}}$, a contradiction. Hence $P=P_{\alpha}$ for some $\alpha \in A$, and thus $R^{*_{\alpha}}=R_{P_{\alpha}}=R_{P}$. So $I^{*_{A}}=I R^{*_{\alpha}}$ for each $I \in \bar{F}(R)$. (3) $\Rightarrow(1)$ : This follows from Corollary 4 .

When we have a standard, countable descending chain of finite type and stable semistar operations over a valuation domain, we can say a little more:

Corollary 5. Let $R$ be a valuation domain and $\mathcal{C}=\left\{*_{i}\right\}_{i=0}^{\infty}$ a standard, countable descending chain of finite type and stable semistar operations on $R$. Then the following are equivalent.

(1) $*_{c}$ is finite type.

(2) $\nu_{\mathcal{C}}$ has finite range.

(3) $\cap_{n \geq 0} G(\nu, n)$ is a localizing system of $R$ of finite type.

(4) $\cap_{n \geq 0} G(\nu, n)=G(\nu, m)$ for some $m \in \mathbb{N}$.

Proof. (1) $\Leftrightarrow(2)$ : Note that $I \in G\left(\nu_{\mathcal{C}}, n\right)$ if and only if $\nu_{\mathcal{C}}(I) \geq n$ if and only if $I^{*_{n}}=R^{*_{n}}$ if and only if $I \in \mathcal{F}^{*_{n}}$. Thus the conclusion follows from Lemma 20 and Lemma 16, (2) $\Rightarrow(4)$ : If the range of $\nu_{\mathcal{C}}$ is finite, then there is $m \geq 0$ with $G\left(\nu_{\mathcal{C}}, n\right)=G\left(\nu_{\mathcal{C}}, m\right)$ for every $n \geq m$. Since $\left\{G\left(\nu_{\mathcal{C}}, n\right)\right\}_{n>0}$ is a descending chain of localizing systems, the claim holds. $(4) \Rightarrow(3)$ : This follows from the fact that $G\left(\nu_{\mathcal{C}}, n\right)$ is of finite type for each $n \geq 0$. (1) $\Leftrightarrow(3)$ : Follows from Theorem 6 . 


\section{REFERENCES}

1. D.D. Anderson, Star operations induced by overrings, Comm. Alg. 16(12) (1988), 2535-2553.

2. N. Bourbaki, Algebré Commutative, Hermann, Paris, 1961-1965.

3. S. Chapman and S. Glaz, One Hundred Problems in Commutative Ring Theory, Non-Noetherian commutative ring theory, 459-476, Math. Appl., 520, Kluwer Acad. Publ., Dordrecht, 2000.

4. M. Fontana and J. A. Huckaba, Localizing systems and Semistar operations, Non-Noetherian commutative ring theory, 169-197, Math. Appl., 520, Kluwer Acad. Publ., Dordrecht, 2000.

5. M. Fontana, J. A. Huckaba and I. J. Papick, Prüfer domains, Marcel Dekker, New York, 1997.

6. M. Fontana, P. Jara and E. Santos, Prüfer *-multiplication Domains, Journal of Algebra and Its Applications, 2(1) (2003), 21-50.

7. M. Fontana and K. A. Loper, Kronecker function rings; a general approach, Ideal theoretic methods in commutative algebra, D.D. Anderson and I.J. Papick, eds., Lecture Notes in Pure and Applied Mathematics, 220, 189-205, Marcel Dekker, Inc., New York, 2001.

8. M. Fontana and G. Picozza, Semistar invertibility on integral domains, Algebra Colloqium, 12(4) (2005), 645-664.

9. J. Garcia, P. Jara and E. Santos, Prüfer *-multiplication domains and torsion theories, Comm. Alg., 27(3) (1999), 12751295.

10. R. Gilmer, Multiplicative Ideal Theory, Marcel Dekker, New York, 1972.

11. Irving Kaplansky, Commutative Rings, Allyn and Bacon, New York, 1970.

12. W. Krull, Idealtheorie, Springer Verlag, Berlin, 1935.

13. Marley, T. A note on rings for which finitely generated ideals have only finitely many minimal components, available on: arXiv:math/0601566 2006.

14. R. Matsuda and A. Okabe, Semistar-operations on integral domains, Math. J. Toyama Univ., 17 (1994), $1-21$.

15. Hideyuki Matsumura, Commutative Ring Theory, Cambridge University Press, Cambridge, 1986.

16. W. Fanggui and R.L. McCasland, On w-modules over strong Mori domains, Comm. Alg., 25(4) (1997), $1285-1306$.

17. A. Mimouni, Semistar operations of finite character on integral domains, Journal of Pure and Applied Algebra 200 (2005), 37-50.

18. Northcott, D.G., Finite Free Resolutions, Cambridge Tracts in Mathematics no 71, Cambridge, Cambridge University Press, 1976.

19. G. Picozza, Semistar Operations and Multiplicative Ideal Theory, Dissertation, Universit'a degli Studi Roma Tre, June 2004.

20. G. Picozza, Star operations on Overrings and Semistar operations, Comm. Alg., 33(6) (2005), 2051-2073.

21. G. Picozza and F. Tartarone, When the semistar operation $\widetilde{*}$ is the identity, Comm. Alg., 36(5) (2008), $1954-1975$.

22. B. Stenström, Rings of Quotients, Springer, Berlin, 1975.

23. M. Zafrullah, The v-operation and intersection of quotient of integral domains, Comm. Alg., 13(8) (1985), 1699-1712.

Department of Mathematics, Glendale Community College, Glendale, California 91208 U.S.A.

Email address: hyunc@glendale.edu

Department of Mathematics, University of California, Riverside, Riverside, California 92521 U.S.A.

Email address: tmcel001@ucr.edu

Department of Mathematics, Whittier College, Whittier, California 90608 U.S.A.

Email address: awalker3@whittier.edu 